III. Aus der Poliklinik für Hals- und Nasenkrankheiten von Prof. Dr. H. Krause in Berlin.

\title{
Ein Fall von Endothelioma myxomatodes palati mollis.
}

Von Dr. H. Cordes, I. Assistenten der Poliklinik.

Unter den Geschwülsten, welche am weichen Gaumen beobachtet werden, nehmen diejenigen Tumoren eine besondere Stellung ein, welche sich zwischen den beiden Blättern des weichen Gaumens entwickeln. Mikulicz ${ }^{1}$ ) bezeichnet dieselben als „intramurale Geschwülste“ und will unter diesem Namen abgekapselte Fibrome, Myxome, Lipome, Chondrome, Endotheliome, wahre Adenome und Mischgeschwülste, wie sie in dieser Gegend zur Entwickelung kommen, verstanden wissen.

Als Entstehungsursache der eben genannten Tumoren glaubt Mikulicz, gleich wie König, für die Geschwülste der Speicheldrüsen eine congenitale Anlage annehmen zu müssen, da einmal zweifellos im embryonalen Leben entstandene Geschwülste hier vorkommen, andererseits der complicirte Bau der meisten die Vermuthung nahe legt, dass Entwickelungsstörungen bei ihrer Entstehung mitspielen. Die complicirten entwickelungsgeschichtlichen Vorgänge bei der Bildung des Gaumens, die so häufig zu Spalt- und Missbildungen desselben führen, lassen die Idee, dass hier auch leicht Versprengungen von Gewebselementen im Sinne Cohnheim's, die nachher zu wuchern beginnen, stattfinden können, als sehr plausibel erscheinen.

In neuerer Zeit hat sich besonders nach den Arbeiten von Volkmann ${ }^{2}$ ) und Eisenmenger ${ }^{3}$ ) die Ansicht Bahn gebrochen, dass die allergrösste Mehrzahl der hierher gehörenden Tumoren zu den Endothelgeschwülsten zu zählen sind. Dieselben haben nach Mikulicz am weichen und harten Gaumen ihren Lieblingssitz und stellen fast für die Gaumengegend typische Geschwulstformen dar.

Die Gaumengeschwülste sind im ganzen recht seltene Tumoren. Volkmann ${ }^{2}$ ) konnte unter Benutzung der gesammten

l) Mikulicz, Neubildungen des Rachens und des Nasenrachenraumes IV. Handbuch der Laryngologie und Rhinologie von Heymann Bd. II, S. 353 u. ff.

$\left.{ }^{2}\right)$ R. Volkmann, Ueber endotheliale Geschwülste, zugleich ein Beitrag zu den Speicheldrüsen- und Gaumentumoren. Deutsche Zeitschrift für Chirurgie 1895, Bd. XLI, S. $1 \mathrm{ff}$.

3) V. Eisenmenger, Ueber die plexiformen Sarkome des harten und weichen Gaumens und deren Stellung zu den anderen dort vorkommenden Geschwülsten. Deutsche Zeitschrift für Chirurgie 1894, Bd. XXXIX, S. 1 u. f. 
Litteratur 138 Fälle zusammenstellen. Unter diesen fanden sich nach der von den betreffenden Autoren meist auf Grund mikroskopischer Untersuchung gestellten Diagnose: Fibrome $11 \mathrm{mal}$, Lipome $2 \mathrm{mal}$, Schleimpolypen $1 \mathrm{mal}$, Cysten $2 \mathrm{mal}$, papilläres Angiom $1 \mathrm{mal}$, Angioma cavernosum $3 \mathrm{mal}$, Rhabdomyom $1 \mathrm{mal}$, Fungus (?) 3 mal, Enchondrome 9 mal, Mischtumoren 5 mal, aberrirte Mandel $1 \mathrm{mal}$, Adenome $32 \mathrm{mal}$, Sarkome $25 \mathrm{mal}$, Epitheliome $3 \mathrm{mal}$, Carcinome $24 \mathrm{mal}$, Cylindrome $2 \mathrm{mal}$. Nur $4 \mathrm{mal}$ wurden bisher Endotheliome durch die mikroskopische Untersuchung nachgewiesen.

Für die Klärung der Morphologie der Gaumengeschwülste erschien es mir daher nicht unwichtig zu sein, die Casuistik um einen genau beobachteten und mikroskopisch untersuchten Fall dieser Art zu bereichern.

Der nachstehend beschriebene Fall stammt aus der Privatpraxis des Herrn Prof. Krause. Ich hatte Gelegenheit, denselben mit zu beobachten und mikroskopisch zu untersuchen.

Bevor ich jedoch den Fall beschreibe, möchte ich noch vorweg bemerken, dass man unter Endotheliom eine Geschwulst versteht, die durch eine atypische Wucherung del platten, endothelialen Zellen entsteht, die die Spalträume, Lymph- und Blutgefässe oder serösen Höhlen auskleiden, oder derjenigen Zellen, die als platter, perivasculärer Belag den Blutcapillaren anliegen. Für die mikroskopische Diagnostik muss die Entstehung der Geschwulst durch Wucherung endothelialer Zellen direkt ersichtlich oder wenigstens nachweisbar sein, dass die Vergrösserung durch endotheliale Wucherung erfolgt (Volkmann).

Ein etwas mannigfaltiges Aussehen bekommen diese Tumoren dadurch, dass in ihnen eine starke Neigung zu degenerativen Veränderungen besteht. Es findet sich colloide, hyaline und schleimige Metamorphose fast stets an einzelnen Stellen oder über die ganze Geschwulst verbreitet. An dieser Veränderung können sich sowohl das bindegewebige Stützgerïst, die Gefässe, sowie auch die Geschwulstzellen betheiligen. Dadurch wird das mikroskopische Bild oft ein schwer zu deutendes und recht mannigfaltiges.

Die klinischen Erscheinungen sind sehr gering und treten meist erst bei beträchtlicher Grösse der Tumoren auf. So kann eine Geschwulst unter Umständen jahrzehntelang bestehen, ohne dass der Träger eine Ahnung von ihrer Existenz hat. Oft wird dieselbe dann erst durch einen Zufall, wie auch im nachstehenden Falle, entdeckt. Die grösseren Tumoren sind auch nicht schmerzhaft, sie verursachen schliesslich nur Schling- und Schluckbeschwerden und machen Sprachstörungen, eventuell durch Druck auf die Epiglottis Athemnoth.

Die Tumoren sind im ganzen gutartig, machen fast nie $\mathrm{Me}$ tastasen, und wenn man sie rechtzeitig exstirpirt, pflegt fast nie ein Recidiv einzutreten.

G. T., 34 Jahre. Patient, ein kräftig gebauter, gesund aussehender Mann, klagt über Schmerzen im Halse, Schluckbeschwerden und Behinderung beim Sprechen seit etwa 14 Tagen. Aus der Anamnese ist folgendes hervorzuheben:

Im Jahre 1883 acquirirte Patient Lues, die durch eine Schmierkur beseitigt wurde. 1884 Recidiv, wiederum Schmierkur. 1888 erkrankte Patient an schmerzhafter Anschwellung im Halse, die durch Eisumschläge in einigen Tagen geheilt wurde. Sonst will Patient immer gesund gewesen sein.

Eine Schwester des Patienten erkrankte vor mehreren Jahren an einem Gallmengeschwiir, welches zu einer Perforation des harten Gaumens führte, so dass die Patientin einen Obturator tragen muss.

Patient bemerkte zuerst bei Gelegenheit einer Zahnextraction vor etwa drei Wochen eine etwa fingergliedgrosse, schmerzlose Anschwellung an der linken Seite des Gaumens, die sich von der Mittellinie und oberhalb des Zäpfchens nach links hin erstreckte. Dieselbe hatte bisher absolut keine Beschwerden verursacht. Nach Angabe des Patienten fühlte sich die Geschwulst etwas hart elastisch an.

Patient suchte nun einen Arzt auf, der Gurgelungen verordnete. Am andern Tage versuchte derselbe angeblich die Geschwulst mit Daumen und Zeigefinger auszudrücken. Die Manipulation war schmerzhaft. Die Geschwulst vergrösserte sich bis zum nächsten Tage sehr stark und erreichte schliesslich seine jetzige Grösse, d. h. etwa die Grösse eines halben Hühnereies. Es trat dauernde Schmerzhaftigkeit ein, sodass Patient einige Tage seinem Berufe fern bleiben musste. Innerlich wurde KJ. genommen. Da der Zustand sich nicht änderte und die Geschwulst sich nicht verkleinerte, riethen die behandelnden Aerzte dem Patienten, nach Berlin zu fahren.

Befund am 12. April 1900: Beim Oeffnen des Mundes bemerkt man eine etwa halbhühnereigrosse, flach halbkugelige Geschwulst von glatter Oberfläche, welche die linke Hälfte des weichen Gaumens einnimmt.
Dieselbe wird von der ganz normalen Schleimhaut straff überzogen und fühlt sich prall elastisch an. Am Halse ist keine Drüsenschwellung bemerkbar.

Durch eine vorgenommene Punction wird etwas Blut herausbefördert. Es handelt sich also um einen compacten Tumor.

Die Differentialdiagnose schwankt wegen der anamnestisch festgestellten Lues zwischen einem Syphilom und einem intramuralen Tumor. Gegen Syphilom spricht jedoch der Mangel einer entzündlichen Röthung der Schleimhaut über dem Tumor und in der Umgebung desselben, sowie die Lage in einer Seitenhälfte des Gaumens, die für die abgekapselten Geschwülste die Regel bildet, während die Syphilome erfahrungsgemäss in der Medianlinie zu liegen pflegen.

Therapie: Spaltung der Schleimhaut und Exstirpation des Tumors. Auskratzung zurückgebliebener Reste mit dem scharfen Löffel. Tamponade.

Die exstirpirten Geschwulstmassen sind von zäher Consistenz und haben ein roth und graugelb gesprenkeltes Anssehen. Auf Druck entleeren sich aus den Gewebsmassen graue Pfröpfe.

Die mikroskopische Untersuchung der in üblicher Weise gehärteten und eingebetteten Geschwulst ergiebt folgenden Befund:

Der Tumor ist zusammengesetzt aus einem bindegewebigen $\mathrm{Ge}$ rüste, welches myxomatöse und hyaline Veränderungen anfweist, und darin eingelagerte Zellmassen. Die hyaline und myxomatöse Degeneration des Bindegewebes ist meist gegenüber den noch fibrösen Partieen iiberwiegend ausgebildet. Das Stroma ist auf weite Strecken hin kernlos und in eine homogene Masse umgewandelt, die sich theils mit Eosin stark rosa, meist mit Hämatoxylin bläulich färbt. Das Verhalten von Hyalin und Mucin bei der Anwendung der Färbung mit Hämatoxylin-Eosin ist derartig, dass sich Hyalin im allgemeinen eosinroth färbt und von dem Hämatoxylin nichts annimmt, während der Schleim blau wird und keine oder nur schwache Eosintinction zeigt. Auf diese Weise ist an den Präparaten die mehr hyaline oder myxomatöse Veranderung aufs deutlichste zu erkennen. Sehr schön treten die schleimig degenerirten Partieen auch bei Anwendung der Thioninfärbung durch Annahme eines röthliclivioletten Farbentones hervor.

In dem bindegewebigen Stroma verlaufen die Gefässe und Capil laren, die jedoch auch zum Theil von der regressiven Veränderung mitergriffen sind.

In den jüngeren Partieen des Tumors ist das Bindegewebe noch out erhalten. An solchen Stellen kann man aufs deutlichste auch die Anfangsstadien der Geschwulst beobachten. In den Spalt-, resp. Lymphräumen des Bindegewebes kommt es zum Wachsthum der endothelialen Zellauskleidung, die zuerst eine einfache Hypertrophie der Endothelzellen darstellt. Dieselben sind grösser, protoplasmareicher und kleiden zuerst in einschichtiger Lage, gleichwie Epithelien angeordnet, den Lymphraum aus (Fig. a). In weiterer Wucherung begriffen wird die

Fig. 84 .

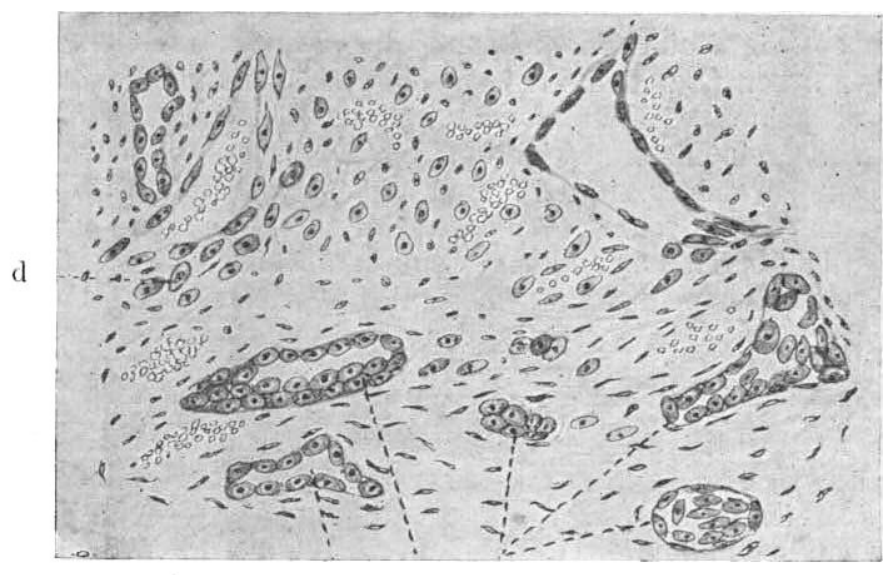

a b

Anordnung der Zellen stellenweise eine mehrschichtige, ein Lumen bleibt, jedoch noch erhalten (Fig. b). Schliesslich verschwindet dann das Limen, und wir haben einen zapfenartigen, in Bindegewebe eingebetteten, soliden Zellstrang vor uns (Fig. c). An anderen Stellen ist die Zellvermehrung stärker. Es kommt zum Auftreten grösserer solider Zellhaufen. In denselben bilden sich bisweilen concentrische Schichtungskugeln, die central hyalin degeneriren, und ferner kleine cystische Hohlräume, sodass dadurch das Bild ein recht complicirtes wird. Solche Stellen haben bisweilen sogar grosse Aehnlichkeit mit Carcinom. Es findet sich jedoch nirgends eine ganz scharfe Abgrenzung zwischen bindegewebigem Stroma und den Geschwulstzellen, wie beim Carcinom. Man kann vielmehr bei genauem Zusehen stets den Zusammenhang der epithelartigen Zellen mit dem Bindegewebe nachweisen.

Die Geschwulstzellen selbst sind der Hauptsache nach platte, rundliche oder unregelmässig geformte, epithelähnliche Elemente mit einem bläschenförmigen Kern, an dem letzteren erkennt man besonders deut- 
lich gefärbt ein oder zwei Kernkörperchen. An manchen Stellen sind die Zellen mehr spindelförmig, der Kern ist länglich oval. Die Anordnung der Zellen ist meist strangartig. Von dem Hauptstrang zweigen sich manchmal kleine Zellzïge ab, die mit einem andern anastomosiren oder stumpf im Bindegewebe der Umgebung endigen. Während die eben geschilderten Wucherungsvorgänge sich offenbar in den Saftspalten und Lymphgefässen abspielen, sieht man an einigen Stellen des Tumors auch eine gleichzeitige Betheiligung der Capillaren, und zwar eine Wucherung der Capillaradventitiazellen. Der Aussenwand der Capillaren liegt eine ein- oder mehrschichtige Kellauflagerung an, die sich vom umgebenden Bindegewebe deutlich durch die Form und Grösse der Zellen unterscheidet. Einzelne dieser Zellen strahlen dann noch weiter in das Bindegewebe aus. Diese Zellen haben meist Spindelform (Fig. d). Die Capillarendothelien selbst erscheinen manchmal auch etwas vergrössert und gequollen, doch sind keine Proliferationserscheinungen nach dem Lumen zu erkennbar.

Nach diesem Befunde dürfte es wohl nicht zweifelhaft sein, dass wir es in dem vorliegenden Fall mit einem typischen Endotheliom zu thun haben, welches seinen Ausgang von den Endothelien der Saftspalten und Lymphgefässe genommen hat und an dessen complicirtem Aufbau sich auch die Adventitia der Blutcapillaren betheiligt hat. Die beobachtete, vorwiegend myxomatöse und hyaline Umwandlung des Stromas, die, wie schon oben erwähnt wurde, ein häufiges Accidens der Endotheliome darstellt, erschwerte die Diagnose insofern, als es erst nach längerem Suchen gelang, Stellen zu finden, die die Genese des Tumors klar erkennen liessen.

Bezüglich des klinischen Verlaufes ist noch zu bemerken, dass Patient am 16. Juni, also nach circa acht Wochen, völlig geheilt entlassen werden konnte. 